\title{
Distinct HLA class I and II genotypes and haplotypes are associated with multiple sclerosis in Bahrain
}

\author{
MOUDI AL-NASHMI ${ }^{1}$, SAFA TAHA ${ }^{1}$, ABDEL HALIM SALEM ${ }^{2}$, ISA ALSHAROQI ${ }^{3}$ and MOIZ BAKHIET ${ }^{1}$ \\ ${ }^{1}$ Department of Molecular Medicine, Princess Al-Jawhara Center for Genetics and Inherited Diseases; \\ ${ }^{2}$ Department of Anatomy, College of Medicine and Medical Sciences, Arabian Gulf University, Manama 329; \\ ${ }^{3}$ Department of Neuroscience, Salmaniya Medical Complex, Manama 12, Bahrain
}

Received May 3, 2018; Accepted October 10, 2018

DOI: $10.3892 /$ br.2018.1164

\begin{abstract}
Multiple sclerosis (MS) has become prevalent in the Arabian Gulf area with high incidence in Bahrain due to environmental influences and genetic susceptibilities, but there is a lack of study into human leukocyte antigen (HLA) types in patients with MS in Bahrain. The present study aimed to study the HLA types expressed in MS patients compared with in control subjects. Blood samples from 50 Bahraini patients with MS and 50 Bahraini control subjects' were subjected to HLA tissue typing by polymerase chain reaction using sequence-specific primers. In comparison with those in control subjects, the allele frequencies of HLA class-I antigens A2, A9, A19, B5, B35 and B40 were higher in MS patients. For class II antigens, the allele frequencies of DR3, DR4 and DR16 were higher in MS patients. The allele frequency of DR15 was lower in MS patients than in control subjects but the difference was not statistically significant $(\mathrm{P}=0.138)$. The higher prevalence of the HLA-ABDR allele was common among the female patients with MS, in relapse remission stage, in cases with higher expanded disability status scale scores and with disease duration between 4 and 9 years. Haplotype HLA-A2-B40-DR2 exhibited significantly higher frequency in MS patients compared with in control subjects $(\mathrm{P}=0.03)$. In conclusion, the results indicated different alleles associated with MS compared with previous reviews. The present study supports the importance of identifying genetic susceptibilities and targets for therapies in specific populations and individuals, to personalize disease management in terms of prediction, protective measures and treatment.
\end{abstract}

Correspondence to: Professor Abdel Halim Salem, Department of Anatomy, College of Medicine and Medical Sciences, Arabian Gulf University, 61 King Abdulaziz Avenue, Al Suqqaya, Manama 329, Bahrain

E-mail: ahaleemfd@agu.edu.bh

Key words: multiple sclerosis, alleles, genes, expanded disability status scale, human leukocyte antigen typing, polymerase chain reaction

\section{Introduction}

Multiple sclerosis (MS) is a severe neurological disorder in which oligodendrocytes are destroyed leading to disability, physical impairment, reduced quality of life and, potentially, mortality (1). MS affects 2.5 million individuals worldwide $(2,3)$. The majority of individuals with MS are in the 20-50-year age range, and the disease tends to affect women 2-3 times more than men $(4,5)$. The main etiology of MS remains largely unknown, though genetics and environmental risk factors are considered to serve a major role in disease pathology (6). The human leukocyte antigen (HLA) complex has been linked with susceptibility to a number of neurodegenerative diseases, including MS (7). Although it is apparent that the HLA region is associated with MS disease risk, the specific genes responsible have been difficult to determine (7). Over 200 genes associated with MS have been identified and, of these, 110 non-HLA genetic loci have been detailed and 13 HLA loci identified $(6,8)$. The haplotype of the HLA-DR15 antigen, DRB1*15:01-DQA1*01:02-DQB1*06:02, has been studied and found to be notably associated with MS, increasing the risk of disease development by 3-fold (9). In the current study the aim was to determine the HLA types expressed in Bahraini patients with MS and compare them with those in control subjects. Additionally, it was determined whether the DRB1*15:01 allele was associated with MS in the Bahraini population studied.

\section{Materials and methods}

Study subjects. Study subjects included 50 Bahraini patients with MS undergoing treatment, who were diagnosed at the Department of Neuroscience of Salmaniya Medical Complex (SMC, Manama, Bahrain). The clinical diagnosis was relapsing remission (RR) in 43 patients, primary progressive (PP) in 4 patients, and secondary progressive (SP) in 3 patients. All MS patients recruited for the study were assessed independently by a neurologist who diagnosed a clinical relapse, in the case of the relapsing cohort, or confirmed clinical remission of the patients in the remission cohort. A magnetic resonance imaging (MRI) brain scan confirmed MS diagnosis according to the McDonald criteria (10). Demographic (age at onset of first symptoms and sex) and clinical (disease course and 
disability status) data, and the findings of all paraclinical tests were recorded for all patients. Disability was evaluated using the Kurtzke expanded disability status scale (EDSS) (11). A total of 50 healthy Bahraini control subjects with no neurological problems or history of autoimmune and inflammatory disease were also enrolled in the study as volunteers, from the SMC Blood Bank.

Inclusion and exclusion criteria included the following: Patients and controls should be of Bahraini origin; patients should be in relapse stage; and healthy control subjects should be without any autoimmune diseases or neurological disorders.

Written informed consent was obtained from all patients under a protocol that was approved by the Research and Ethics Committee of the College of Medicine and Medical Sciences, Arabian Gulf University, Bahrain. Control subjects provided consent on donation of blood samples. Ethical approval from the Ministry of Health, Bahrain was also obtained.

Sample collection and DNA extraction. Blood samples (4 ml) from the MS patients were collected at the Department of Neuroscience, SMC, while control subject samples were collected from the SMC Blood Bank. DNA was extracted from the Buffy coat using a QIAamp ${ }^{\circledR}$ DNA Mini and Blood Mini kit (Qiagen GmbH, Hilden, Germany). The DNA samples' purity was measured with a NanoDrop 1000 spectrophotometer (Thermo Fisher Scientific, Inc., Waltham, MA, USA).

HLA typing. Genotyping of the HLA alleles HLA-A, -B and -DR was performed using polymerase chain reaction sequence-specific primers (PCR-SSP) from Bio-Rad Medical Diagnostics GmbH (Dreieich, Germany). PCR was performed with the HLA SSP series (cat. no. 189738) according to the manufacturer's protocol, as follows: Initial denaturation at $94^{\circ} \mathrm{C}$ for $2 \mathrm{~min}$; 10 cycles of denaturation at $94^{\circ} \mathrm{C}$ for $10 \mathrm{sec}$ and annealing and extension at $65^{\circ} \mathrm{C}$ for $60 \mathrm{sec}$; and 20 cycles of denaturation at $94^{\circ} \mathrm{C}$ for $10 \mathrm{sec}$, annealing at $61^{\circ} \mathrm{C}$ for $50 \mathrm{sec}$ and extension at $72^{\circ} \mathrm{C}$ for $30 \mathrm{sec}$. The PCR products were identified using $2 \%$ agarose gel electrophoresis visualized with ethidium bromide staining. Following completion of the electrophoresis, the gel was placed on a UV transilluminator and photographed for documentation and interpretation.

Statistical analysis. HLA analysis was performed by comparing the dominant allele frequencies and HLA haplotypes between the MS patients and control subjects using Arelquin version 3.5 (http://cmpg.unibe.ch/software/arlequin3/). The high-frequency alleles were analyzed for association with clinical features, by calculating odds ratios (ORs) with $95 \%$ confidence intervals (CIs). Pearson's $\chi^{2}$ and Fisher' exact probability tests were performed on the VassarStats website (http://vassarstats.net/) to analyze differences between the control and MS groups. $\mathrm{P}<0.05$ was considered to indicate statistical significance.

\section{Results}

Demographics and clinical characteristics. HLA-ABDR allelic typing was performed in $50 \mathrm{MS}$ patients (male: female,

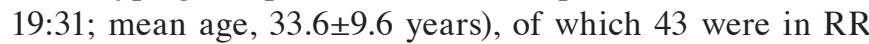
stage, 4 PP stage and 3 SP stage. The analysis also included 50 control subjects (male: female, 47:3; mean age, 38.5 \pm 10.6
Table I. Demographics of the MS patients and control subjects.

\begin{tabular}{|c|c|c|}
\hline & $\begin{array}{l}\text { MS patients } \\
n=50\end{array}$ & $\begin{array}{c}\text { Controls } \\
n=50\end{array}$ \\
\hline \multicolumn{3}{|l|}{ Age, years } \\
\hline Range & $18-56$ & $19-62$ \\
\hline Mean $\pm \mathrm{SD}$ & $33.6 \pm 9.6$ & $38.5 \pm 10.6$ \\
\hline \multicolumn{3}{|l|}{ Sex } \\
\hline Male & 19 & 47 \\
\hline Female & 31 & 3 \\
\hline \multicolumn{3}{|l|}{ EDSS score } \\
\hline Range & $1-8$ & \\
\hline Median & $3.7 \pm 1.4$ & \\
\hline \multicolumn{3}{|c|}{ Disease duration, years } \\
\hline Range & $1-20$ & \\
\hline Mean \pm SD & $6.6 \pm 5.5$ & \\
\hline \multicolumn{3}{|l|}{ MS type } \\
\hline $\mathrm{RR}$ & 43 & \\
\hline $\mathrm{PP}$ & 4 & \\
\hline SP & 3 & \\
\hline \multicolumn{3}{|l|}{ Drug } \\
\hline Avonex & 12 & \\
\hline Betaferon & 9 & \\
\hline Gilenya & 4 & \\
\hline Not available & 6 & \\
\hline Rebif & 17 & \\
\hline Tysabri & 2 & \\
\hline
\end{tabular}

MS, multiple sclerosis; EDSS, expanded disability status scale; $\mathrm{RR}$, relapse remitting; PP, primary progressive; SP, secondary progressive; $\mathrm{SD}$, standard deviation.

years). On evaluating MS progression, the degree of disability indicated by EDSS score was a median of 3.7, and the mean disease duration in terms of symptoms was $6.6 \pm 5.5$ years. The 50 MS patients were undergoing treatment with Avonex, Betaferon, Gilenya, Rebif or Tysabri (12, 9, 4, 6, 17 and 2 patients, respectively; Table I).

HLA-ABDR alleles frequency in MS patients and control subjects. The analysis of HLA-ABDR tissue typing in MS patients detected 9 of HLA-A loci, 23 of HLA-B loci and 9 of HLA-DR loci (Table II). A higher frequency, though not to significant extent, was observed of HLA-A alleles in MS patients compared with in control subjects, namely of A11, A19, A2 and A3. The frequency of the HLA-A9 allele was significantly higher in MS patients compared with in control subjects ( 20 vs. $8 \%$; OR, 2.875; CI, 1.201-6.883; $\mathrm{P}=0.014$ ). However, the HLA-A9 allele did not exhibit significant difference after applying Bonferroni's correction.

A higher frequency, though not significant, of HLA-B alleles was observed in the MS patients compared with in control subjects, namely of B12, B13, B14, B21, B37, B40, B41, B5, B53, B78 and B81. The frequency of the HLA-B35 allele was significantly higher in MS patients compared with 
Table II. Frequencies of HLA-ABDR alleles in MS patients and control subjects.

\begin{tabular}{|c|c|c|c|c|c|c|}
\hline $\begin{array}{l}\text { HLA-ABDR } \\
\text { allele }\end{array}$ & $\begin{array}{c}\text { MS group } \\
\text { frequency, } \%, n=50\end{array}$ & $\begin{array}{l}\text { Control group } \\
\text { frequency, } \%, n=50\end{array}$ & P-value & Odds ratio & $\begin{array}{c}95 \% \text { confidence } \\
\text { interval }\end{array}$ & $\mathrm{Pc}$ \\
\hline A1 & 13 & 16 & 0.548 & 1.275 & $0.578-2.811$ & \\
\hline A10 & 8 & 23 & 0.003 & 3.435 & $1.454-8.114$ & 0.030 \\
\hline A11 & 3 & 2 & 1 & 1.516 & $0.248-9.271$ & \\
\hline A19 & 26 & 20 & 0.312 & 1.405 & $0.724-2.728$ & \\
\hline A 2 & 20 & 17 & 0.583 & 1.221 & $0.597-2.497$ & \\
\hline A 28 & 6 & 9 & 0.420 & 1.549 & $0.530-4.528$ & \\
\hline A3 & 3 & 2 & 1 & 1.516 & $0.248-9.271$ & \\
\hline A36 & 1 & 3 & 0.621 & 3.062 & $0.313-29.949$ & \\
\hline A9 & 20 & 8 & 0.014 & 2.875 & $1.201-6.883$ & 0.126 \\
\hline B12 & 7 & 6 & 0.777 & 1.179 & $0.382-3.641$ & \\
\hline B13 & 2 & 1 & 1 & 2.020 & $0.180-22.646$ & \\
\hline B14 & 5 & 4 & 1 & 1.263 & $0.329-4.849$ & \\
\hline B15 & 5 & 12 & 0.075 & 2.591 & $0.877-7.651$ & \\
\hline B16 & 2 & 6 & 0.279 & 3.128 & $0.616-15.886$ & \\
\hline B17 & 4 & 5 & 1 & 1.263 & $0.329-4.849$ & \\
\hline B18 & 5 & 5 & 1 & 1 & $0.280-3.567$ & \\
\hline B21 & 7 & 4 & 0.350 & 1.807 & $0.512-6.376$ & \\
\hline B22 & 1 & 2 & 1 & 2.020 & $0.180-22.646$ & \\
\hline B27 & 0 & 4 & 0.121 & & & \\
\hline B35 & 12 & 3 & 0.015 & 4.409 & $1.204-16.141$ & 0.345 \\
\hline B37 & 2 & 1 & 1 & 2.020 & $0.180-22.646$ & \\
\hline B40 & 10 & 4 & 0.096 & 2.667 & $0.808-8.807$ & \\
\hline B41 & 2 & 1 & 1 & 2.020 & $0.180-22.646$ & \\
\hline B42 & 1 & 1 & 1 & 1 & $0.0617-16.213$ & \\
\hline B47 & 1 & 1 & 1 & 1 & $0.0617-16.213$ & \\
\hline B5 & 17 & 14 & 0.559 & 1.258 & $0.583-2.715$ & \\
\hline B53 & 2 & 0 & 0.497 & & & \\
\hline B7 & 3 & 6 & 0.497 & 2.064 & $0.502-8.493$ & \\
\hline B73 & 3 & 6 & 0.497 & 2.064 & $0.502-8.493$ & \\
\hline B78 & 5 & 3 & 0.720 & 1.702 & $0.396-7.321$ & \\
\hline B8 & 3 & 11 & 0.026 & 3.996 & $1.079-14.791$ & 0.59 \\
\hline B81 & 1 & 0 & 1 & & & \\
\hline DR1 & 10 & 16 & 0.207 & 1.714 & $0.737-3.988$ & \\
\hline DR2 & 20 & 20 & 1 & 1 & $0.500-1.999$ & \\
\hline DR3 & 17 & 16 & 0.841 & 1.075 & $0.509-2.269$ & \\
\hline DR4 & 16 & 11 & 0.300 & 1.511 & $0.676-3.512$ & \\
\hline DR5 & 15 & 11 & 0.399 & 1.428 & $0.621-3.284$ & \\
\hline DR6 & 7 & 12 & 0.228 & 1.812 & $0.682-4.811$ & \\
\hline DR7 & 14 & 11 & 0.521 & 1.317 & $0.567-3.062$ & \\
\hline DR8 & 0 & 2 & 0.497 & & & \\
\hline DR9 & 1 & 1 & 1 & 1 & $0.062-16.213$ & \\
\hline
\end{tabular}

Allele frequencies are presented as the percentage of each allele in relation to total number of alleles $(2 \mathrm{n})$ in the group. When a single allele was found it was considered homozygous and counted twice. P-values were obtained using Pearson's $\chi^{2}$ or Fisher's exact (two-sided) tests. Pc was for the number of different alleles tested (multiplication of P-values by 9 for HLA-A alleles, by 23 for HLA-B alleles and by 9 for HLA-DR alleles). HLA, human leukocyte antigen; MS, multiple sclerosis; Pc, corrected P-value.

in controls (12 vs. 3\%; OR, 4.409; CI, 1.204-16.141; $\mathrm{P}=0.015$ ). However, the HLA-B35 allele did not show significant difference after applying Bonferroni's correction. A higher frequency, though not significant, of the HLA-DR alleles was revealed in MS patients compared with control subjects, of DR3, DR4, DR5 and DR7.

Conversely, the HLA-A10 allele exhibited significantly higher frequency in control subjects compared with in MS 
Table III. High-frequency HLA-ABDR alleles and clinical features of MS patients.

\begin{tabular}{|c|c|c|c|c|c|c|c|c|c|}
\hline \multirow{2}{*}{$\begin{array}{l}\text { HLA-ABDR } \\
\text { allele }\end{array}$} & \multicolumn{2}{|c|}{ Sex } & \multirow[b]{2}{*}{ P-value } & \multirow{2}{*}{$\begin{array}{c}\text { Age, } \\
\text { mean } \pm \mathrm{SD}\end{array}$} & \multicolumn{3}{|c|}{ MS type } & \multirow{2}{*}{$\begin{array}{l}\text { EDSS score, } \\
\text { mean } \pm \text { SD }\end{array}$} & \multirow{2}{*}{$\begin{array}{l}\text { Disease duration, } \\
\text { mean } \pm \mathrm{SD}\end{array}$} \\
\hline & $\mathrm{F}$ & M & & & $\mathrm{RR}$ & PP & SP & & \\
\hline A19 & 12 & 10 & 0.6315 & $33.8 \pm 9.8$ & 18 & 2 & 2 & $3.5 \pm 0.3$ & $7.7 \pm 7.0$ \\
\hline A2 & 9 & 7 & 0.5838 & $35.0 \pm 1.4$ & 14 & 1 & 1 & $3.6 \pm 1.0$ & $7.2 \pm 5.6$ \\
\hline A9 & 11 & 5 & 0.1016 & $33.0 \pm 7.0$ & 15 & 1 & 0 & $4.0 \pm 1.4$ & $4.8 \pm 4.8$ \\
\hline B35 & 7 & 3 & 0.1821 & $33.6 \pm 12.7$ & 9 & 1 & 0 & $3.2 \pm 1.0$ & $6.4 \pm 7.0$ \\
\hline B5 & 15 & 1 & 0.0001 & $33.1 \pm 9.8$ & 14 & 1 & 1 & $3.7 \pm 0.7$ & $5.6 \pm 0.7$ \\
\hline B40 & 5 & 4 & 1 & $35.6 \pm 8.4$ & 7 & 1 & 1 & $4.0 \pm 0.3$ & $8.0 \pm 7.0$ \\
\hline DR3 & 12 & 5 & 0.0624 & $33.5 \pm 7.7$ & 15 & 0 & 2 & $3.4 \pm 0.7$ & $7.0 \pm 2.8$ \\
\hline DR4 & 6 & 8 & 0.5656 & $36.0 \pm 2.8$ & 12 & 1 & 1 & $3.7 \pm 0.7$ & $8.8 \pm 4.9$ \\
\hline DR5 & 8 & 7 & 0.7772 & $31.0 \pm 2.8$ & 12 & 2 & 1 & $3.7 \pm 1.4$ & $6.7 \pm 5.3$ \\
\hline
\end{tabular}

HLA, human leukocyte antigen; MS, multiple sclerosis; RR, relapse remitting; PP, primary progressive; SP, secondary progressive; EDSS, expanded disability status scale; F, female; M, male; SD, standard deviation.

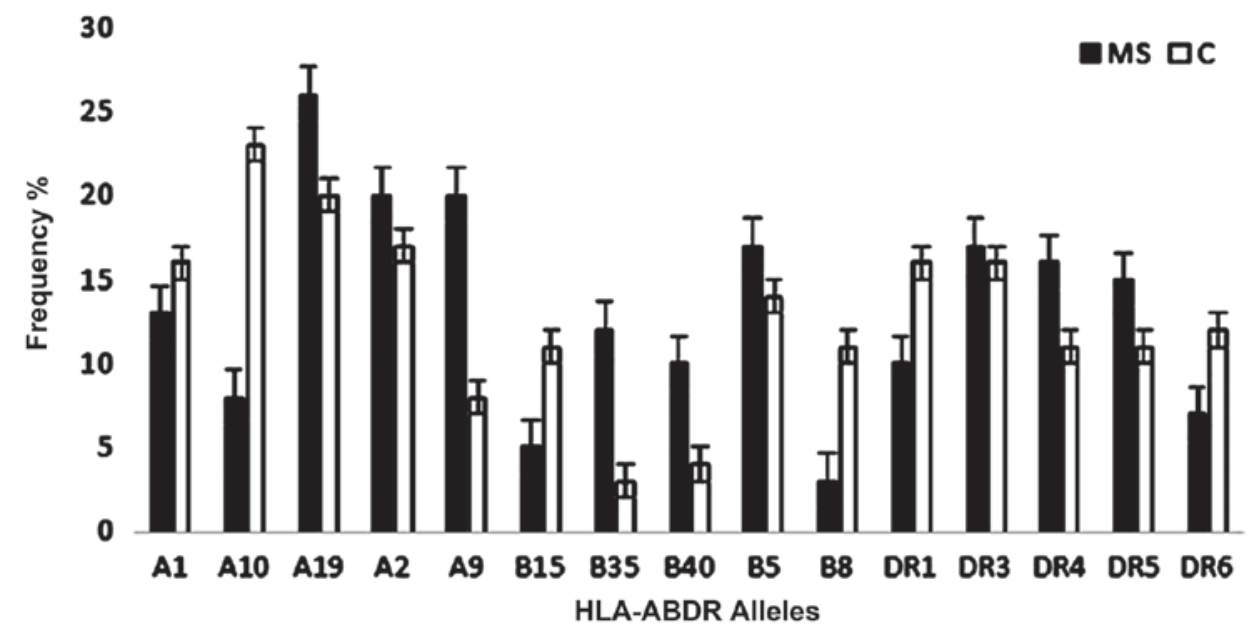

Figure 1. Most frequent HLA-ABDR alleles in MS patients and control subjects. The results revealed that HLA-A19, A9, A2, B5 and DR3 were the highest frequency alleles in MS patients, and HLA-A10 the highest frequency allele in control subjects. Error bars indicate the mean \pm standard error of the mean. HLA, human leukocyte antigen; MS, multiple sclerosis; C, control.

patients (28 vs. $8 \%$; OR, 3.435; CI, 1.454-8.114; $\mathrm{P}=0.003$ ), and HLA-A1 had marginally higher frequency in control subjects than in MS patients (16 vs. 13\%; OR, 1.275; CI, 0.578-2.811; $\mathrm{P}=0.548)$.

Furthermore, HLA-B15 was present at higher frequency in control subjects than in MS patients (12 vs. 5\%; OR, 2.591; CI, 0.877-7.651; $\mathrm{P}=0.075$ ), and HLA-B8 showed significantly higher allele frequency in control subjects than in MS patients (11 vs. 3\%; OR, 3.996; CI, 1.079-14.791; P=0.026); however, HLA-B8 did not exhibit significant difference after applying Bonferroni's correction. Additionally, HLA-DR1 (16 vs. 10\%; OR, 1.714; CI, 0.737-3.988; $\mathrm{P}=0.207)$ and DR6 (12 vs. 7\%; OR, 1.812; CI, 0.682-4.811; $\mathrm{P}=0.228$ ) showed higher allele frequencies in control subjects than in MS patients.

HLA-B18 and HLA-DR2 exhibited equal frequencies between the study populations. HLA-DR15 was present at lower frequency in MS patients than in control subjects (6 vs. 12\%; OR, 2.136; CI, 0.769-5.938) but the difference was not statistically significant $(\mathrm{P}=0.138$; Table VI). Fig. 1 presents the most frequent HLA-ABDR alleles detected in MS patients and control subjects. Figs. 2 and 3 show representative gel electrophoresis results of PCR products for the HLA loci in an MS patient and a healthy control subject.

Association of high-frequency HLA-ABDR alleles with MS clinical features. HLA alleles A19, A2, A9, B35, B5, B40, DR3 and DR5 were more common in female MS patients, while HLA-DR4 was more common in male MS patients. HLA-B5 exhibited significantly higher allele frequency in female patients than in male patients $(\mathrm{P}=0.001)$. Regarding disease progression, these alleles were notably more frequent in the RR stage of MS. The high-frequency HLA-ABDR alleles of MS patients also appeared associated with EDSS scores between 3 and 4, and with disease duration ranging from 4 to 9 years (Table III).

High-frequency allele subtypes of MS patients and control subjects. Table IV presents the high-frequency allele subtypes 


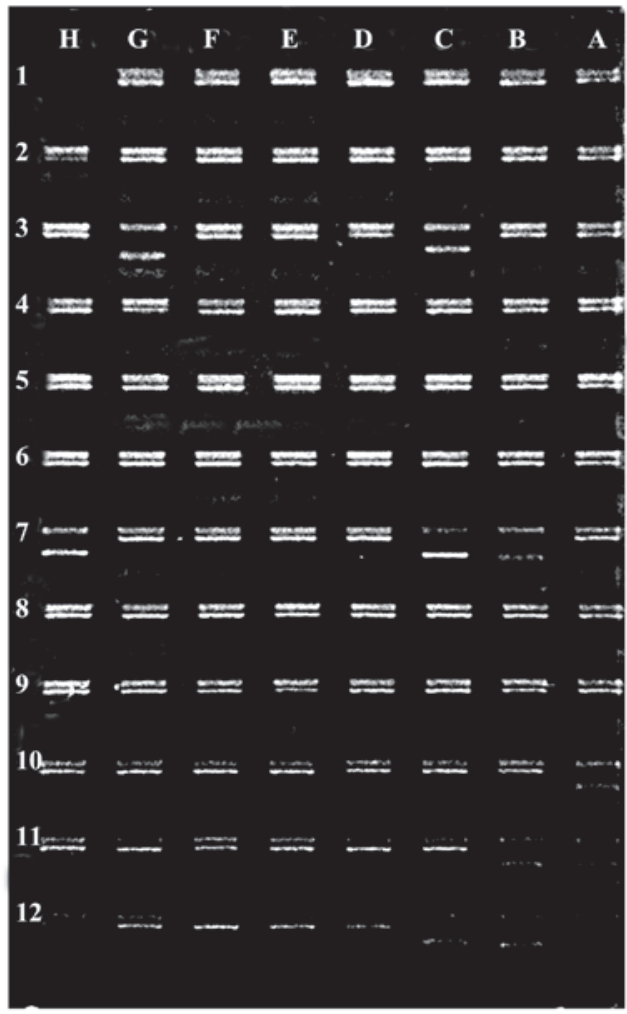

Figure 2. Example electrophoresis gel of PCR products for HLA loci in a patient with multiple sclerosis. Negative control (position H1), HLA-A25 (position H2), HLA-A32 (position C3), HLA-A32 (position G3), HLA-B50 (position B7), HLA-B50 (position C7), HLA-B44 (position H7), HLA-DR4 (position A10), HLA-DR13 (position B11), HLA-DR13 (position B11), HLA-DR53 (position B12), HLA-DR52 (position C12) and HLA-DR13 (position H12). HLA, human leukocyte antigen.

of HLA-ABDR in MS patients compared with control subjects. Regarding HLA-A genotypes; HLA-A19, and HLA-A9 were higher in MS patients compared to control subjects. The HLA-A30 allele was more frequent in MS patients compared with in control subjects ( 8 vs. 3\%; $\mathrm{P}=0.120)$. HLA-A24 was significantly higher in MS patients than in controls even following Bonferroni correction (16 vs. 5\%; $\mathrm{P}=0.02$ ). HLA-DR11 showed higher frequency in MS patients than in control subjects ( 15 vs. $11 \% ; \mathrm{P}=0.399$ ).

Table V reports the high-frequency allele subtypes of HLA-ABDR in control subjects compared with MS patients. HLA-A26 exhibited higher allele frequency in control subjects compared with in MS patients (12 vs. $5 \%$; $\mathrm{P}=0.075$ ), as well as HLA-DR13 (10 vs. 5\%; $\mathrm{P}=0.161$ ).

Table VI depicts the subtypes of alleles with equal frequency between the study populations.

Comparison between HLA-ABDR haplotypes of MS patients and control subjects. HLA-ABDR haplotypes in patients and controls were analyzed. Only haplotypes present in at least $2 \%$ of the sample were considered. This identified 90 prevalent haplotypes (data not shown) in MS patients and control subjects. The haplotype HLA-A2-B40-DR2 exhibited significantly higher frequency in MS patients compared with in controls ( 5 vs. $0 \%$; $\mathrm{P}=0.030$ ). Meanwhile, 4 haplotypes were common between MS patients and control subjects: HLA-A1-B5-DR3, HLA-A10-B12-DR4, HLA-A19-B15-DR7

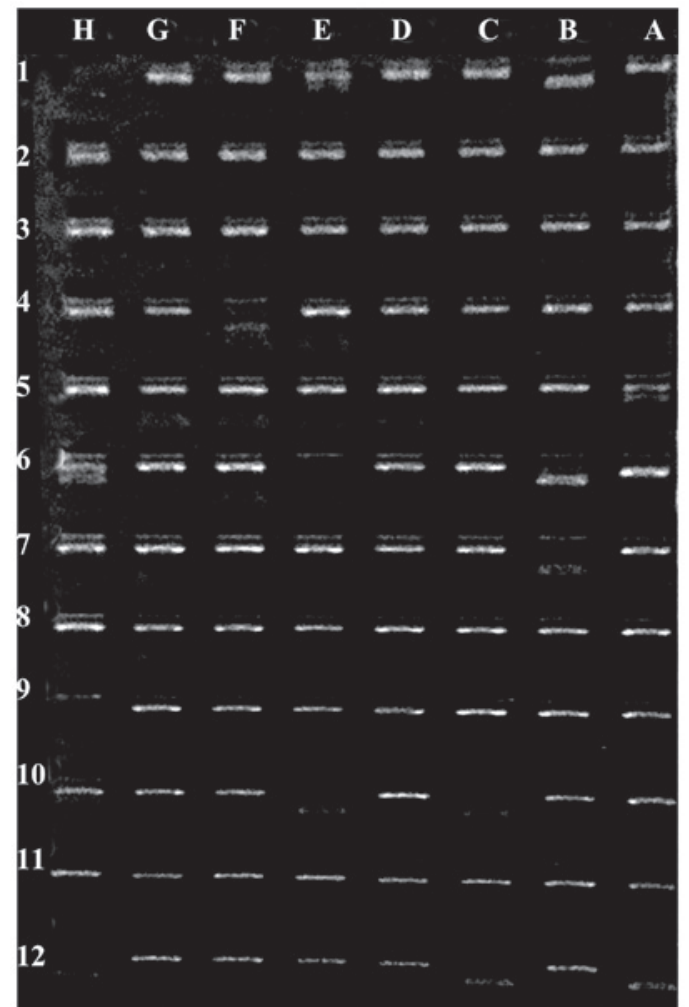

Figure 3. Example electrophoresis gel of PCR products for HLA loci in a healthy control subject. Negative control (position H1), HLA-A24 (position B1), HLA-A3 (position E1), HLA-B8 (position F4), HLA-B8 (position A5), HLA-B8 (position A6), HLA-B47 (position B6), HLA-B8 (position H6), HLA-B47 (position B7), HLA-DR17 (position C10), HLA-DR15 (position E10), HLA-Bw6 (position H10), HLA-DR51 (position A12), HLA-DR52 (position C12) and HLADR17 (position H12). HLA, human leukocyte antigen.

and HLA-A9-B5-DR3. Among these, 2 showed the same frequency, HLA-A1-B5-DR3 and HLA-A19-B15-DR7 (2\%); the other 2 (HLA-A10-B12-DR4 and HLA-A9-B5-DR3) were slightly higher in MS patients (3 vs. $2 \%$ ). Overall a total of 10 haplotypes exhibited higher frequencies in MS patients than in controls. Meanwhile, 3 haplotypes (HLA-A2-B15-DR3, HLA-A2-B15-DR7 and HLA-A19-B15-DR1) were present only in control subjects (Table VII).

\section{Discussion}

MS is a complex multifactorial disease caused by interaction between environmental and genetics risk factors (12). There have been numerous reports of increased prevalence of MS worldwide in the last 5 years, with the Arabian Gulf area exhibiting a moderate-to-high prevalence (31-55 MS cases per 100,000 individuals) (13). Over 400 MS cases were identified in the neuroscience department at SMC (unpublished data). The present study aimed to assess the genetic risk factors among HLA loci that may be involved in the pathogenesis of MS in Bahraini patients.

The importance of HLA class I and II in the risk of developing MS has been widely studied (14). Several studies from the United States (15) have confirmed the association of the HLA class II alleles DRB1*15:01, DQA1*01:02 and DQB1*06:02 with MS, while other studies mapped HLA class II DRB $5 * 01: 01$, DRB1*15:01, DQA1*01:02 and DQB1*06:02 alleles in the North 
Table IV. High-frequency allele subtypes of MS patients vs. control subjects.

\begin{tabular}{|c|c|c|c|c|c|c|}
\hline $\begin{array}{l}\text { HLA-ABDR } \\
\text { allele }\end{array}$ & $\begin{array}{c}\text { MS group } \\
\text { frequency, } \%, n=50\end{array}$ & $\begin{array}{c}\text { Control group } \\
\text { frequency, } \%, n=50\end{array}$ & P-value & Odds ratio & $\begin{array}{l}95 \% \text { confidence } \\
\text { interval }\end{array}$ & $\mathrm{Pc}$ \\
\hline \multicolumn{7}{|l|}{ A19 } \\
\hline A29 & 4 & 6 & 0.516 & 1.532 & $0.419-5.603$ & \\
\hline A30 & 8 & 3 & 0.120 & 2.812 & $0.724-10.924$ & \\
\hline $\mathrm{A} 31$ & 2 & 0 & 0.490 & & & \\
\hline A32 & 9 & 9 & 1 & 1 & $0.379-2.634$ & \\
\hline A33 & 3 & 2 & 1 & 1.516 & $0.248-9.271$ & \\
\hline A2 & 20 & 17 & 0.583 & 1.221 & $0.597-2.497$ & \\
\hline \multicolumn{7}{|l|}{ A9 } \\
\hline A23 & 4 & 3 & 0.999 & 1.347 & $0.294-6.180$ & \\
\hline A24 & 16 & 5 & 0.011 & 3.619 & $1.271-10.303$ & 0.022 \\
\hline B35 & 12 & 3 & 0.015 & 4.409 & $1.204-16.141$ & 0.345 \\
\hline B40 & 7 & 3 & 0.195 & 2.434 & $0.611-9.694$ & \\
\hline B60 & 1 & 0 & 1 & & & \\
\hline B61 & 2 & 1 & 1 & 2.020 & $0.180-22.646$ & \\
\hline \multicolumn{7}{|c|}{ B5 } \\
\hline B51 & 14 & 7 & 0.106 & 2.163 & $0.834-5.612$ & \\
\hline B52 & 3 & 7 & 0.194 & 2.434 & $0.611-9.694$ & \\
\hline \multicolumn{7}{|l|}{ DR3 } \\
\hline DR17 & 15 & 15 & 1 & 1 & $0.460-2.173$ & \\
\hline DR18 & 2 & 1 & 1 & 2.020 & $0.180-22.646$ & \\
\hline DR4 & 16 & 11 & 0.300 & 1.541 & $0.676-3.512$ & \\
\hline \multicolumn{7}{|l|}{ DR5 } \\
\hline DR11 & 15 & 11 & 0.399 & 1.428 & $0.621-3.284$ & \\
\hline
\end{tabular}

HLA, human leukocyte antigen; MS, multiple sclerosis; Pc, corrected P-value.

European population $(16,17)$. Other alleles may contribute to MS in European populations, in particular HLA-DQA1*05:01, HLA-B*44:02 and HLA-C*05 (18). In Sardinian MS patients, disease pathology has been associated with the DRB1*03:01, DQA1*05:01, DQB1*02:01 and DRB1*04:05, DQA1*05:01 and DQB1*03:01 alleles (17,19). In African-Brazilian MS patients, the strongest association was observed with DQB1*06:02 (19). In Canadian MS families, the DRB1*15:01 allele appeared to determine susceptibility to MS (19).

In the present study, the relative frequencies of HLA class I and II alleles and haplotypes were assessed in 50 Bahraini patients with MS. Overall, current observations are not in line with a previous reports (20), which has concluded the highest risk HLA allele associated with MS to be HLA-DRB1*15:0, and that for HLA haplotype to be HLA-DRB1*15:01-HLA-DQA1*01:02-HLA-DQB1*06:02-

HLA-DRB5*01:01 (20).

The present results suggested other susceptibility alleles of HLA-ABDR genotypes, in particular HLA-A9 and B35. In addition, HLA-ABDR allele subtypes A24 was higher in MS patients compared with in controls. This finding is similar to results of a study in Kuwaiti patients with MS disease (21). Additionally, the current results on HLA-A9 and A19 were similar to findings reported by Chao et al (22). Furthermore, the higher frequency observed of HLA-A2 in MS patients is similar to previous reports across populations from The Netherlands, Switzerland, USA and Scandinavia $(20,23)$. Our result on HLA-A24 is further in the line with an Iranian study (24). Moreover, the findings on HLA-DR3 are similar to those reported in a Russian Altai territory population (25). Regarding HLA-B5 and DR4, the present results were similar to an Iraqi study that reported association of these alleles with MS; however the same study indicated the protective alleles of HLA-B35 and DR2, which differs from present findings (26). Previous studies have suggested that the HLA-A2 allele has a protective effect in MS (27,28). The current study further indicated a positive association and therefore protective effect of HLA-A10 allele in healthy control subjects compared with MS patients, similar to a study by Amirzargar et al (29). By contrast, Al-Shammri et al (21) and Chao et al (22) found that HLA-A10 was higher in MS patients. The finding that HLA-B8 and B15 may serve as protective alleles also differs to previous results of Chao et al (22) and Jilek et al (28). Regarding HLA-DR6 as a protective allele, the current data is in line with the Kuwaiti study while differing from findings in Japan and Mexico (21,30). The indication of HLA-DR13 as a protective allele is similar to a Spanish study (31) but dissimilar to an Italian study (32). 
Table V. High-frequency allele subtypes of control subjects vs. MS patients.

\begin{tabular}{|c|c|c|c|c|c|c|}
\hline $\begin{array}{l}\text { HLA-ABDR } \\
\text { allele }\end{array}$ & $\begin{array}{c}\text { Control group } \\
\text { frequency, } \%, n=50\end{array}$ & $\begin{array}{c}\text { MS group } \\
\text { frequency, } \%, n=50\end{array}$ & P-value & Odds ratio & $\begin{array}{l}95 \% \text { confidence } \\
\text { interval }\end{array}$ & $\mathrm{Pc}$ \\
\hline A1 & 16 & 13 & 0.548 & 1.275 & $0.578-0.811$ & \\
\hline A10 & 7 & 2 & 0.169 & 3.688 & $0.746-18.211$ & \\
\hline A25 & 0 & 1 & 1 & & & \\
\hline A26 & 12 & 5 & 0.075 & 2.591 & $0.877-7.651$ & \\
\hline A34 & 1 & 0 & 0.999 & & & \\
\hline A43 & 2 & 0 & 0.490 & & & \\
\hline A66 & 1 & 0 & 0.999 & & & \\
\hline B15 & 12 & 5 & 0.075 & 2.591 & $0.877-7.651$ & \\
\hline B62 & 0 & 0 & 0 & & & \\
\hline B63 & 0 & 0 & 0 & & & \\
\hline B70 & 0 & 0 & 0 & & & \\
\hline B71 & 0 & 0 & 0 & & & \\
\hline B72 & 0 & 0 & 0 & & & \\
\hline B75 & 0 & 0 & 0 & & & \\
\hline B76 & 0 & 0 & 0 & & & \\
\hline B77 & 0 & 0 & 0 & & & \\
\hline B8 & 11 & 3 & 0.026 & 3.996 & $1.079-14.791$ & 0.590 \\
\hline DR1 & 16 & 10 & 0.207 & 1.714 & $0.737-3.988$ & \\
\hline DR6 & 12 & 7 & 0.228 & 1.812 & $0.68-4.811$ & \\
\hline DR13 & 10 & 5 & 0.161 & 2.250 & $0.709-7.141$ & \\
\hline DR14 & 2 & 2 & 1 & 1 & $0.135-7.392$ & \\
\hline
\end{tabular}

HLA, human leukocyte antigen; MS, multiple sclerosis; Pc, corrected P-value.

Table VI. HLA-ABDR alleles of equal frequency between MS patients and control subjects.

\begin{tabular}{|c|c|c|c|c|c|}
\hline $\begin{array}{l}\text { HLA-ABDR } \\
\text { allele }\end{array}$ & $\begin{array}{c}\text { MS group } \\
\text { frequency, } \%, n=50\end{array}$ & $\begin{array}{c}\text { Control group } \\
\text { frequency, } \%, n=50\end{array}$ & P-value & Odds ratio & $\begin{array}{c}95 \% \text { confidence } \\
\text { interval }\end{array}$ \\
\hline B18 & 5 & 5 & 1 & 1 & $0.280-3.567$ \\
\hline DR2 & 20 & 20 & 1 & 1 & $0.500-1.999$ \\
\hline DR15 & 6 & 12 & 0.138 & 2.136 & $0.769-5.938$ \\
\hline DR16 & 14 & 8 & 0.174 & 0.534 & $0.214-1.337$ \\
\hline
\end{tabular}

HLA, human leukocyte antigen; MS, multiple sclerosis.

The current result on HLA-DR15 is dissimilar to findings in the Russian Altai study, which indicated association with a high risk of MS (25). HLA-DR2 exhibited an equal allele frequency between the current study populations, similar to a US study (33).

The current study further indicated HLA-A19, A2, A9, B35, B5, B40, DR5 and DR3 were found to be more common in female MS patients, in RR stage, in MS patients, in cases with EDSS scores between 3 and 4, and in cases with disease duration between 4 and 9 years. Notably, HLA-B5 was common and significantly higher $(\mathrm{P}=0.0001)$ in female compared with male patients, whereas HLA-DR4 was more common in male patients in RR stage, and was observed in patients above 30 years old, similar to a study performed in Qatar (13). The results on HLA-DR2 and DR4 were similar to the Kuwaiti study. In contrast, a US study and others suggested have that DR2 is apparent in several forms of MS (34). Although the HLA-DRB1*15 haplotype may represent the main disease risk factor in populations of North European origin, several dissimilar allelic associations have been identified in Southern European populations, Israel (35) and other countries (30). The comparison of HLA-ABDR haplotypes between MS and control subjects determined 10 haplotypes with higher frequency in MS and absence in control subjects. A total of 5 haplotypes carried HLA-A19, 4 carried HLA-B5 and 5 carried HLA-DR2, indicating susceptibility alleles. 
Table VII. HLA haplotypes of MS patients and control subjects.

\begin{tabular}{|c|c|c|c|c|c|c|c|c|}
\hline \multirow{2}{*}{$\begin{array}{l}\text { HLA-ABDR } \\
\text { allele }\end{array}$} & \multicolumn{3}{|c|}{ HLA haplotype } & \multirow{2}{*}{$\begin{array}{l}\text { MS group } \\
\text { frequency, } \% \\
n=50\end{array}$} & \multirow{2}{*}{$\begin{array}{c}\text { Control group } \\
\text { frequency, } \% \\
n=50\end{array}$} & \multirow[b]{2}{*}{$\mathrm{P}$-value } & \multirow{2}{*}{$\begin{array}{l}\text { Odds } \\
\text { ratio }\end{array}$} & \multirow{2}{*}{$\begin{array}{c}95 \% \\
\text { confidence } \\
\text { interval }\end{array}$} \\
\hline & A & B & DR & & & & & \\
\hline 1 & A1 & B5 & DR3 & 2 & 2 & 1 & 0.993 & $0.139-7.098$ \\
\hline 2 & A19 & B15 & DR7 & 2 & 2 & 1 & 0.993 & $0.139-7.098$ \\
\hline 3 & A9 & B5 & DR3 & 3 & 2 & 0.686 & 0.660 & $0.109-3.979$ \\
\hline 4 & A10 & B12 & DR4 & 3 & 2 & 0.686 & 0.660 & $0.109-3.979$ \\
\hline 5 & A1 & B5 & $\mathrm{DR} 2$ & 3 & 0 & 0.123 & & \\
\hline 6 & A19 & B35 & DR2 & 3 & 0 & 0.123 & & \\
\hline 7 & A19 & B40 & $\mathrm{DR} 2$ & 3 & 0 & 0.123 & & \\
\hline 8 & A19 & B5 & DR4 & 3 & 0 & 0.123 & & \\
\hline 9 & A19 & B14 & DR7 & 3 & 0 & 0.123 & & \\
\hline 10 & $\mathrm{~A} 2$ & B35 & DR2 & 3 & 0 & 0.123 & & \\
\hline 13 & A9 & B18 & DR5 & 3 & 0 & 0.123 & & \\
\hline 14 & A19 & B5 & DR3 & 4 & 0 & 0.061 & & \\
\hline 15 & $\mathrm{~A} 2$ & B5 & DR4 & 4 & 0 & 0.061 & & \\
\hline 16 & A2 & B40 & DR2 & 5 & 0 & 0.030 & & \\
\hline 17 & A2 & B15 & DR3 & 0 & 3 & 0.249 & & \\
\hline 18 & $\mathrm{~A} 2$ & B15 & DR7 & 0 & 3 & 0.249 & & \\
\hline 19 & A19 & B15 & DR1 & 0 & 4 & 0.124 & & \\
\hline
\end{tabular}

HLA, human leukocyte antigen; MS, multiple sclerosis .

A significant positive association of HLA-A2-B40-DR2 was detected in the Bahraini MS patients $(\mathrm{P}=0.030)$. Therefore, the most potent genetic effects appear to be conferred by A2 and DR2, which is similar to findings in several previous studies $(9,19,23)$.

Interestingly, HLA-A9 and B35 were indicated as risk factor alleles for MS disease, whereas HLA-A10 and B8 were determined as protective alleles. An obvious association of a specific haplotype with a particular autoimmune disease in an ethnic group may not hold in another ethnic group, where haplotypes associations may be differ entirely. Nevertheless, the present results indicate that HLA-A2-B40-DR2 haplotype is significantly higher in Bahraini MS patients when compared with healthy individuals.

In conclusion, observations of different allele and haplotypes associations with MS between various populations emphasizes that there may be a population-specific genetic susceptibility or protection conferred by HLA alleles for MS in different populations reported. HLA-DRB1*1501 has been consistently associated with MS susceptibility in genetic studies. The current study did not find evidence of association between HLA-DRB1*1501 and MS in the Bahraini patients studied. Certain HLA-ABDR antigens (A19, A2, A9, B35, B40, B5, DR3, DR4 and DR5) were present at higher frequencies in the MS Bahraini patients compared with controls; while HLA-A10 was indicated as a protective allele, being detected at higher frequency in the control subjects. The current indications of HLA allele associations with MS differ from observations in Caucasians and certain Arab populations, though are similar to findings in Kuwaiti patients.

\section{Acknowledgements}

The authors acknowledge the Bahrain Multiple Sclerosis Patients Society for their assistance in recruiting patients to the study.

\section{Funding}

The present study was supported by a grant (no. 76) from the Research Committee of the College of Medicine and Medical Sciences, Arabian Gulf University, Manama, Bahrain.

\section{Availability of data and materials}

The datasets used and/or analyzed during the current study are available from the corresponding author on reasonable request.

\section{Authors' contributions}

MAN performed the experiments. ST contributed to the acquisition, analysis and interpretation of data. AHS contributed to the statistical analysis and writing of the paper. IA performed the clinical aspects of the study. MB is the principle investigator of the study and wrote the paper.

\section{Ethics approval and consent to participate}

The study was approved by the Ethics Committee of the College of Medicine and Medical Sciences, Arabian Gulf University and the Ethical Committee of the Ministry of 
Health, Bahrain. Written informed consent from all subjects agreeing to their participation and use of samples in the current study was obtained.

\section{Patient consent for publication}

Consent was obtained from the participants agreeing to the publication of any associated data.

\section{Competing interests}

The authors declare that they have no competing interests.

\section{References}

1. Rhead B, Bäärnhielm M, Gianfrancesco M, Mok A, Shao X, Quach H, Shen L, Schaefer C, Link J, Gyllenberg A, et al: Mendelian randomization shows a causal effect of low vitamin D on multiple sclerosis risk. Neurol Genet 2: e97, 2016.

2. Sahraian MA, Sahebkar M, Dehghani R, Derakhshan-Jazari M, Kazami-Moghaddam V and Kouchaki E: Multiple sclerosis-A disease on a dramatically rising trend in Iran: Review of possible reasons. Iran J Neurol 16: 34-40, 2017.

3. Dargahi N, Katsara M, Tselios T, Androutsou ME, de Courten M, Matsoukas J and Apostolopoulos V: Multiple sclerosis: Immunopathology and treatment update. Brain Sci 7: E78, 2017.

4. Harbo HF, Gold R and Tintoré M: Sex and gender issues in multiple sclerosis. Ther Adv Neurol Disorder 6: 237-248, 2013.

5. Johnson KM, Zhou H, Lin F, Ko JJ and Herrera V: Real-world adherence and persistence to oral disease-modifying therapies in multiple sclerosis patients over 1 year. J Manag Care Spec Pharm 23: 844-852, 2017.

6. Mescheriakova JY, Broer L, Wahedi S, Uitterlinden AG, van Duijn CM and Hintzen RQ: Burden of genetic risk variants in multiple sclerosis families in the Netherlands. Mult Scler J Exp Transl Clin 2: 2055217316648721, 2016.

7. Fernando MM, Stevens CR, Walsh EC, De Jager PL, Goyette P, Plenge RM, Vyse TJ and Rioux JD: Defining the role of the MHC in autoimmunity: A review and pooled analysis. PLoS Genet 4 e1000024, 2008

8. Moutsianas L, Jostins L, Beecham AH, Dilthey AT, Xifara DK, Ban M, Shah TS, Patsopoulos NA, Alfredsson L, Anderson CA, et al; International IBD Genetics Consortium (IIBDGC): Class II HLA interactions modulate genetic risk for multiple sclerosis. Nat Genet 47: 1107-1113, 2015.

9. Parnell GP and Booth DR: The multiple sclerosis (MS) genetic risk factors indicate both acquired and innate immune cell subsets contribute to MS pathogenesis and identify novel therapeutic opportunities. Front Immunol 8: 425, 2017.

10. Polman CH, Reingold SC, Banwell B, Clanet M, Cohen JA, Filippi M, Fujihara K, Havrdova E, Hutchinson M, Kappos L, et al: Diagnostic criteria for multiple sclerosis: 2010 revisions to the McDonald criteria. Ann Neurol 69: 292-302, 2011

11. Institute of Medicine (US) Committee on Multiple Sclerosis: Current Status and Strategies for the Future. In: Multiple Sclerosis. Joy JE and Johnston RB Jr (eds). National Academies Press, Washington, DC, 2001

12. Moosazadeh M, Esmaeili R, Mehdi Nasehi M, Abedi G, Afshari M,Farshidi F and Kheradmand M: Prevalence of familial multiple sclerosis in Iran: A systematic review and meta-analysis. Iran J Neurol 16: 90-95, 2017.

13. Deleu D, Mir D, Al Tabouki A, Mesraoua R, Mesraoua B, Akhtar N, Al Hail H, D'souza A, Melikyan G, Imam YZ, et al: Prevalence, demographics and clinical characteristics of multiple sclerosis in Qatar. Mult Scler 19: 816-819, 2013.

14. Link J, Lundkvist Ryner M, Fink K, Hermanrud C, Lima I, Brynedal B, Kockum I, Hillert J and Fogdell-Hahn A: Human leukocyte antigen genes and interferon beta preparations influence risk of developing neutralizing anti-drug antibodies in multiple sclerosis. PLoS One 9: e90479, 2014.

15. Hebbring SJ, Schrodi SJ, Ye Z, Zhou Z, Page D and Brilliant MH: A PheWAS approach in studying HLA-DRB1*1501. Genes Immun 14: 187-191, 2013.
16. Anagnostouli MC, Acquaviva T, Artemiadis AK, Rentzos M, Karandreas N, Davaki P and Stamboulis E: HLA-DRB1* alleles genotyping in chronic inflammatory demyelinating polyneuropathy in Greek patients. J Peripher Nerv Syst 19: 187-189, 2014.

17. Cocco E, Sardu C, Pieroni E, Valentini M, Murru R, Costa G, Tranquilli S, Frau J, Coghe G, Carboni N, et al: HLA-DRB1-DQB1 haplotypes confer susceptibility and resistance to multiple sclerosis in Sardinia. PLoS One 7: e33972, 2012.

18. Healy BC, Liguori M, Tran D, Chitnis T, Glanz B, Wolfish C, Gauthier S, Buckle G, Houtchens M, Stazzone L, et al: HLA B*44: Protective effects in MS susceptibility and MRI outcome measures. Neurology 75: 634-640, 2010.

19. Alcina A, Abad-Grau MM, Fedetz M, Izquierdo G, Lucas M, Fernández O, Ndagire D, Catalá-Rabasa A, Ruiz A, Gayán J, et al: Multiple sclerosis risk variant HLA-DRB1*1501 associates with high expression of DRB1 gene in different human populations. PLoS One 7: e29819, 2012.

20. Patsopoulos NA, Barcellos LF, Hintzen RQ, Schaefer C, van Duijn CM, Noble JA, Raj T, Gourraud PA, Stranger BE, Oksenberg J, et al; IMSGC; ANZgene: Fine-mapping the genetic association of the major histocompatibility complex in multiple sclerosis: HLA and non-HLA effects. PLoS Genet 9: e1003926, 2013.

21. Al-Shammri S, Nelson RF, Al-Muzairi I and Akanji AO: HLA determinants of susceptibility to multiple sclerosis in an Arabian Gulf population. Mult Scler 10: 381-386, 2004.

22. Chao MJ, Barnardo MC, Lincoln MR, Ramagopalan SV, Herrera BM, Dyment DA, Montpetit A, Sadovnick AD, Knight JC and Ebers GC: HLA class I alleles tag HLA-DRB1*1501 haplotypes for differential risk in multiple sclerosis susceptibility. Proc Natl Acad Sci USA 105: 13069-13074, 2008.

23. Link J, Kockum I, Lorentzen AR, Lie BA, Celius EG, Westerlind H, Schaffer M, Alfredsson L, Olsson T, Brynedal B, et al: Importance of human leukocyte antigen (HLA) class I and II alleles on the risk of multiple sclerosis. PLoS One 7: e36779, 2012.

24. Kalanie H, Kamgooyan M, Sadeghian H and Kalanie AR: Histocompatibility antigen (HLA) associations with multiple sclerosis in Iran. Mult Scler 6: 317-319, 2000.

25. Smagina IV, El'chaninova SA, Zolovkina AG, Ignatova IuN and Kudriavtseva EA: Genetic risk factors for multiple sclerosis in the population of Altay. Zh Nevrol Psikhiatr Im S S Korsakova 111: 42-45, 2011 (In Russian)

26. Saleem MA, Mukhelif HF, Moussawi KM and Al-Khafaji JT: Human leukocyte antigen typing in Iraqi multiple sclerosis patients. Neurosciences (Riyadh) 12: 127-132, 2007.

27. Gough SC and Simmonds MJ: The HLA region and autoimmune disease: Associations and mechanisms of action. Curr Genomics 8: 453-465, 2007.

28. Jilek S, Schluep M, Harari A, Canales M, Lysandropoulos A, Zekeridou A,Pantaleo G and Du Pasquier RA: HLA-B7-restricted EBV-specific $\mathrm{CD}^{+} \mathrm{T}$ cells are dysregulated in multiple sclerosis. J Immunol 188: 4671-4680, 2012.

29. Amirzargar AA, Tabasi A, Khosravi F, Kheradvar A, Rezaei N, Naroueynejad M, Ansaripour B, Moradi B and Nikbin B: Optic neuritis, multiple sclerosis and human leukocyte antigen: Results of a 4-year follow-up study. Eur J Neurol 12: 25-30, 2005.

30. Hillert J: Human leukocyte antigen studies in multiple sclerosis. Ann Neurol 36 (Suppl 1): S15-S17, 1994.

31. Uría DF: HLA and multiple sclerosis. Studies of a spanish population. Rev Neurol 31: 1066-1070, 2000.

32. Perini P, Tagliaferri C, Belloni M, Biasi G and Gallo P: The HLA-DR 13 haplotype is associated with 'benign' multiple sclerosis in northeast Italy. Neurology 57: 158-159, 2001

33. Barcellos LF, Oksenberg JR, Begovich AB, Martin ER, Schmidt S, Vittinghoff E, Goodin DS, Pelletier D, Lincoln RR, Bucher P, et al; Multiple Sclerosis Genetics Group: HLA-DR2 dose effect on susceptibility to multiple sclerosis and influence on disease course. Am J Hum Genet 72: 710-716, 2003.

34. Vasconcelos CC, Fernández O, Leyva L, Thuler LC and Alvarenga RM: Does the DRB1*1501 allele confer more severe and faster progression in primary progressive multiple sclerosis patients? HLA in primary progressive multiple sclerosis. J Neuroimmunol 214: 101-103, 2009.

35. Schmidt H, Williamson D and Ashley-Koch A: HLA-DR15 haplotype and multiple sclerosis: A HuGE review. Am J Epidemiol 165: 1097-1109, 2007. 